\title{
A TENTATIVE SKETCH FOR BL LACERTAE OBJECTS
}

\author{
HÉLĖNE SOL and LOURDES VICENTE \\ Observatoire de Paris-Meudon, France
}

VLBI polarization data show magnetic field configuration parallel to the nuclear jet in quasars and perpendicular to it in BL Lac objects [1]. It appears difficult to account for this contrast within the unified scheme for AGN [2]. To investigate a direct explanation of this peculiarity of BL Lac objects, we study the possibilities of propagation and radiation of beams of particles in transverse ambient magnetic field. High energy streams with kinetic energy density larger than the ambient magnetic one, $E_{k i n}>E_{\text {mag }}$, can easily propagate with enhancement of the transverse magnetic field at the leading edge of the stream and reconnection of magnetic lines in its wake. Synchrotron radiation in front shocks naturally leads to the observed polarization. Moreover self-polarization, with formation of charge layers and $E \times B$ drift velocity, allows substantial propagation for even lower energy streams with $E_{k i n}<E_{\text {mag }}$, as long as their density $n_{o}$ is large enough, typically $\kappa=4 \pi n_{o} m_{i} c^{2} / B^{2}>1$. Such low energy streams are non diamagnetic and do not modify the ambient field. Any high energy tail of the total particle distribution in the jet therefore radiates in the transverse field pattern. This concerns for instance the BL Lac object $\mathrm{W}$ Comae if we assume a proton-electron jet with bulk velocity $v_{o}=0.1 c$ (as the source does not require relativistic beaming so far), an equipartition magnetic field $\mathrm{B}=0.02 \mathrm{G}$ and a density of radiating particles $n_{r}=0.05 \mathrm{~cm}^{-3}$ at about $7 \mathrm{pc}$ from the nucleus (knot K3). For $n_{r} / n_{o}=10^{-3}$, one gets the stream density $n_{o}=50 \mathrm{~cm}^{-3}$ which allows good propagation as $\kappa$ reaches $2 \times 10^{3}$, and still corresponds to a moderate mass outflow of $0.06 \mathrm{M} \odot$ /year for a VLBI jet cross-sectional area of $2 p c^{2}$.

If the ambient magnetic field is roughly aligned with the large scale jet direction, as observed in stellar jets and expected in the case of a large magnetic structure which favors formation of the accretion disc in the plane perpendicular to the field, we reach the idea that BL Lac objects are active galactic nuclei with highly twisted accretion discs. VLBI beams are emitted from the inner edges of the disc along the central black hole rotation axis while the extended radio source comes from the outer misaligned part of the disc driven by the recently accreted matter. This scenario has several consequences such as the possibility of different orientation of the ionizing cone which can strongly modify the observed spectral lines properties and the occurence of large misalignment angles between parsec and kiloparsec radio structures as often observed in BL Lac objects.

\section{References}

1. Gabuzda, D.C., Cawthorne, T.V., Roberts, D.H., Wardle, J.F.C., 1992, ApJ, 388, p.40

2. Gopal-Krishna, Wiita, P.J., 1993, Nature, 363, p.142

T. J.-L. Courvoisier and A. Blecha: Multi-Wavelength Continuum Emission of AGN, 473. (C) 1994 IAU. Printed in the Netherlands. 